\title{
Cárie dentária em crianças de 5 anos: fatores sociodemográficos, lócus de controle e atitudes parentais
}

\author{
Dental decay in 5-year-old children: sociodemographic factors, \\ locus of control and parental attitudes
}

Vinícius Humberto Nunes ${ }^{1}$

Gimol Benzaquen Perosa ${ }^{2}$

${ }^{1}$ Faculdade de Odontologia, Universidade Estadual de Londrina. Rod. Celso Garcia Cid Km 380, Campus Universitário. 86057-970

Londrina PR Brasil. nunesvh@hotmail.com

${ }^{2}$ Faculdade de Medicina de Botucatu, Universidade Estadual de São Paulo. Botucatu SP Brasil.
Abstract Dental decay affects many children, especially those from the lower socioeconomic classes. In this cross-sectional study designed to investigate the role played by sociodemographic factors, parental attitudes, and locus of control, which are an indicator of personal perception of what controls individual health, on the prevalence of tooth decay among 5-year-old pre-school children living in a midsized city in São Paulo, Brazil. The ceo-d index of 426 children was assessed; the parents reported sociodemographic characteristics and completed two questionnaires concerning locus of control and parental attitudes. The results show that $52.35 \%$ of the children had decay; higher levels of severe decay were observed among lower E-F socioeconomic classes. Higher socioeconomic status and low externality appear to be protective factors. Low parental internality emerged as a risk factor for decay in primary teeth, possibly because the mother expects or delegates the action to others, delaying care. Parental perceptions of control over a child's health seem to impact preventive care and, consequently, the level of tooth decay among children.

Key words Dental decay, Oral health, Child care, Child health
Resumo A cárie dentária afeta grande parte das crianças, principalmente as de baixo nível socioeconômico. Neste estudo, de delineamento transversal, objetivou-se investigar o papel de fatores sociodemográficos, atitudes parentais e do Lócus de Controle, indicador da percepção pessoal sobre o que controla a saúde do sujeito, na prevalência de cárie em pré-escolares de cinco anos, moradores de cidade de médio porte paulista. Avaliou-se o indice ceo-d de 426 crianças; os pais informaram sobre as características sociodemográficas, responderam a dois questionários de Lócus de Controle e um de atitudes parentais. Os resultados mostraram que 52,35\% dos pré-escolares apresentaram cárie, com a severa em níveis mais altos nos estratos mais baixos E-F. Nível socioeconômico mais alto e baixa externalidade mostraram-se como fatores de proteção. Baixa internalidade parental apareceu como um fator de risco para cárie nos dentes decíduos, possivelmente porque a mãe espera ou delega a ação a outros, retardando os cuidados. A percepção parental de controle sobre a saúde do filho parece favorecer cuidados preventivos $e$, consequentemente, o nível de cárie da criança. Palavras-chave Cárie dentária, Saúde bucal, Cuidado da criança, Saúde da criança 


\section{Introdução}

A cárie dentária afeta grande parte da população infantil, é a doença crônica mais comum em crianças $^{1} e$, devido à sua elevada prevalência e aos graves impactos na qualidade de vida e desenvolvimento é considerada um problema de saúde pública². A dor causada pela cárie interfere no ato de comer e faz com que as crianças apresentem crescimento mais lento, mais baixo peso e mais distúrbios no sono ${ }^{3-5}$; prejudica o rendimento escolar com sensível diminuição na atenção durante as atividades, gerando déficit de aprendizagem $^{6}$. Especificamente com relação à dentição decídua, apesar de dados de prevalência muito diversos, observa-se um aumento significativo de cárie à medida que aumenta a idade ${ }^{7}$. No Brasil, segundo a Pesquisa Nacional de Saúde Bucal, SB Brasil $2010^{7}, 53,4 \%$ das crianças apresentavam cárie aos cinco anos, com uma prevalência menor na região sudeste. No Estado de São Paulo, pesquisas realizadas em três cidades do interior paulista (Araraquara, Paulínia e Piracicaba), com crianças com idade entre 4 e 6 anos, mostraram uma prevalência de cárie em torno de $50 \%^{8-10}$.

Se um grande número de pesquisas atribui o surgimento da cárie à associação das variáveis biológicas e dietéticas, nos últimos tempos cresceu o interesse em pesquisar o papel de variáveis sociodemográficas, psicossociais e ambientais ${ }^{11,12}$. Constatou-se uma maior prevalência de cárie em grupos de baixa renda ${ }^{13-15}$, possivelmente pela influência de tipos de dieta alimentar ${ }^{16,17}$, pior nível de autocuidado e baixa procura de atendimento preventivo ${ }^{2,18,19}$, piores condições de higiene bucal $^{20}$ e dificuldade de acesso a serviços odontológicos para a população mais pobre ${ }^{21}$. Vários indicadores têm sido utilizados para medir o nível socioeconômico ${ }^{2,17,22-26}$, no entanto, devido às limitações no uso exclusivo de cada um desses indicadores, Koslowski ${ }^{27}$ e Meneghim et al. ${ }^{28}$ propuseram uma fórmula que considera vários fatores associados (renda, escolaridade, atividade profissional paterna, posse da habitação e número de habitantes por moradia), criando uma hierarquia de seis classes sociais.

Em relação ao papel que variáveis psicossociais desempenham no processo saúde/doença, a literatura destaca a capacidade materna de cuidar como um fator de proteção que amenizaria o impacto de um ambiente desfavorável, especialmente durante os períodos de maior vulnerabilidade da criança, como nos primeiros anos de vida, durante a vigência de uma doença ou quando a família está passando por uma crise finan- ceira $^{29,30}$. A adequação da mãe como cuidadora mostrou estreita relação com sua escolaridade, estado físico, saúde mental, disponibilidade de tempo e a possibilidade de contar com ajuda nos cuidados $^{31}$. Outros estudos deram atenção especial às crenças e valores paternos, principalmente, a percepção que os pais têm a respeito de sua responsabilidade sobre sua saúde e do filho, como uma variável diretamente vinculada ao modo como ela age e se comporta, identificada como Lócus de Controle ${ }^{32,33}$.

O Lócus de Controle, conceito baseado na Teoria da Aprendizagem Social de Bandura ${ }^{34}$, seria um indicador da percepção pessoal sobre quem ou o que controla a determinação de eventos na vida. Indivíduos cuja orientação do Lócus é interna acreditam que eles próprios podem exercer algum controle sobre os acontecimentos; indivíduos cuja orientação é externa atribuem a outros o controle do que ocorre em sua vida - pessoas, entidades, destino, acaso ou mesmo a sorte ${ }^{2,32} . \mathrm{Na}$ área da saúde, sujeitos com predomínio de Lócus de Controle interno apresentaram comportamentos mais adequados, tanto curativos quanto preventivos ${ }^{35}$ e uma tendência a apresentar hábitos bucais mais saudáveis ${ }^{13,14,36}$. Os trabalhos que associam o Lócus de Controle paterno e saúde bucal dos filhos ainda são escassos, com resultados inconclusivos ${ }^{37}$. Chase et $a l .^{38}$ observaram que mães com Lócus de Controle externo tinham filhos com maior risco de desenvolver cárie dentária, enquanto que as de maior internalidade retornavam mais vezes às consultas de controle. Lencová et al. ${ }^{19}$ identificaram uma associação positiva entre internalidade da mãe e menor índice de cárie nos filhos, diferindo de estudo anterior de Brandão et al. ${ }^{2}$.

Nas três pesquisas, entretanto, avaliou-se o controle que os cuidadores tinham sobre sua própria saúde, associando-o ao nível de cárie dos filhos, quando seria mais adequado utilizar uma avaliação especifica de Lócus de Controle parental. Na literatura, identificaram-se três escalas com esse propósito, nenhuma delas traduzida para o português. No Brasil, Cerqueira e Nascimento $^{32}$, com o objetivo inicial de adaptar para o contexto brasileiro um dos instrumentos mais recorrentes na literatura internacional, a escala de Wallston et al. ${ }^{39}$, criaram a Escala de Lócus de Controle Parental na Saúde (LOCPS). Entretanto, ao longo do processo, sentiram a necessidade de criar novos itens, gerando um novo instrumento que se mostrou discriminativo, com índices de precisão entre 0,67 e 0,80.

Além do Lócus de Controle, há pesquisas que associam as atitudes parentais aos hábitos bucais 
dos filhos. Dumitrescu et al. ${ }^{40}$ observaram que atitudes favoráveis contribuíam para a prevenção de cárie e doenças periodontais em pessoas de todas as idades. Com crianças, Navas et al. ${ }^{41}$ identificaram que apesar da grande maioria dos pais explicitar atitudes favoráveis, aqueles que, na prática, apresentavam atitudes inadequadas tinham, de forma significativa, mais filhos com cárie.

Os resultados inconclusivos, a escassez de pesquisas sobre o Lócus de Controle e atitudes parentais na saúde bucal, motivaram esta pesquisa. Procurou-se, avaliar, em crianças com 5 anos, a associação entre o índice de cárie com variáveis socioeconômicas e Lócus de Controle paterno e identificar possíveis fatores de risco e proteção à cárie. Formularam-se as seguintes hipóteses: a) as crianças de 5 anos provenientes dos níveis socioeconômicos mais baixos devem ter maior índice de cárie, quando comparadas com as dos níveis socioeconômicos mais altos; b) crianças de cinco anos, cujos pais têm baixa internalidade parental devem ter maior índice de cárie, quando comparadas com crianças de pais com alta internalidade parental.

\section{Material e Métodos}

O presente estudo, de delineamento transversal, contou com a aprovação do Comitê de Ética em Pesquisa da FMB-UNESP. Os responsáveis pelas crianças que aceitaram participar assinaram o Termo de Consentimento Livre e Esclarecido.

\section{Local do Estudo}

O estudo foi desenvolvido em Avaré, cidade de médio porte do estado de São Paulo que, segundo o IBGE, contava com 82.934 habitantes no censo de $2010^{42}$. A economia gira em torno da agricultura, cítricos e cana de açúcar, pecuária, serviços e turismo. Da população acima de 10 anos, 93,50\% estava alfabetizada e para as crianças em idade pré-escolar, em 2011, a cidade contava com 11 Centros de Educação Infantil (CEI) municipais, 2 sob administração filantrópica e 6 particulares. Nenhuma das entidades municipais contava com cuidado especializado em saúde bucal e dentre as particulares, somente uma escola tem atendimento odontológico.

\section{Participantes}

Dos 2.162 pré-escolares matriculadas nas CEIs, optou-se por estudar somente crianças com cinco anos de idade completos, idade índice adotada pela OMS para inquéritos epidemiológicos ${ }^{43}$, que correspondeu a 531 crianças. Nas escolas privadas, estavam matriculadas 92 crianças com 5 anos. Foram excluídas crianças que haviam sofrido possíveis acidentes, perdas dentárias prematuras, agenesia dental e tivessem feito ou fizessem uso de aparelhos ortodônticos fixos. Os pais foram convidados a participar da pesquisa ao irem buscar os filhos nas Reuniões de Pais e Mestres e demais encontros (festas juninas, dia das mães, etc.), de acordo com a dinâmica de cada instituição. Para os que tinham dificuldade de comparecer nessas ocasiões, foram agendados encontros individuais, em momentos escolhidos por eles. Todos os que aceitaram participar assinaram o "Termo de Consentimento Livre e Esclarecido" e responderam aos questionários, na instituição. Somente alunos cujos pais preencheram os formulários foram avaliados quanto à saúde bucal.

A amostra final, de conveniência, foi composta por 426 pré-escolares com 5 anos, 389 das 439 matriculadas em escolas municipais $(80,22 \%)$ e 37 crianças das 92 inscritas nas escolas particulares (40,2\%). Grande parte das perdas deveu-se a pais que não conseguiram ser localizados ou se recusaram a participar, especialmente das escolas privadas, sendo que 2 escolas não permitiram que o pesquisador entrasse em contato com os pais.

\section{Instrumentos de avaliação}

Para avaliação da saúde bucal das crianças foi utilizado o índice ceo-d, expressão quantitativa da prevalência de cárie em dentes decíduos, obtido pela soma de dentes decíduos cariados, extraídos ou com indicação de extração por lesão de cárie e obturados ${ }^{43}$, usando-se um espelho bucal plano $n^{\circ} 5$ e sonda odontológica nº 1 , sem realização de profilaxia prévia.

\section{Atitude dos pais em relação à higiene bucal}

Aplicou-se um questionário que aborda conhecimentos dos pais em relação ao seu papel nos cuidados dentários, desde o nascimento da criança, assim como frequência e forma de escovação e a relação de cuidado dentário com a saúde geral, elaborado por Navas et al. ${ }^{41}$. Os pais se posicionavam a respeito das 16 afirmativas, a partir de uma escala tipo Likert, com 5 opções que variavam de: Concordo totalmente (1) a Discordo totalmente (5). Portanto, cada afirmativa recebeu um valor de 1 a 5 e a pontuação final 
correspondeu à somatória dos 16 itens ponderados. Considera-se que quanto maior a pontuação, mais acertada a atitude dos pais em relação à saúde bucal dos filhos.

\section{Lócus de controle}

Utilizou-se a Escala Multidimensional de Lócus de Controle da Saúde (MHLC) de Wallston et al. ${ }^{39}$, traduzida, adaptada por Dela Coleta ${ }^{44}$, que obteve uma consistência interna de 0,54 e uma confiabilidade de 0,50, para amostras brasileiras. A escala MHLC apresenta três subescalas, cada uma com seis itens referentes às dimensões: internalidade no Lócus de Controle (ILC), extensão na qual se acredita que fatores internos do indivíduo determinam o estado de saúde/doença; externalidade no Lócus de Controle (ELC), crença na qual a saúde/doença é determinada por outros (Deus, profissionais da saúde, etc.); acaso no Lócus de Controle (ALC), crença de que a saúde/doença é determinada pelo acaso, sorte ou destino.

As respostas às 18 afirmações, são dadas a partir de uma escala tipo Lickert de 5 pontos, variando de "Concordo Totalmente" (1) a "Discordo Totalmente" (5). Os escores por indivíduo podem variar de 6 a 30 pontos para cada subescala (I, P e C), sendo que, quanto menor o escore obtido na subescala, maior a concordância com aquele Lócus, ou seja, maior a crença que aquele fator controla a saúde.

\section{Lócus de Controle adaptado}

Aplicou-se a Escala Multidimensional do Lócus de Controle da Saúde (MHLC), como descrito acima, mas desta vez mudava-se a pergunta e pedia-se ao sujeito que se posicionasse em relação ao Lócus de Controle da saúde do filho (Ex: "Não importa o que EU faça, sempre é possível que MEU FILHO fique doente"). A forma de avaliação das respostas e a análise dos resultados foi a mesma da adotada na escala original.

\section{Avaliação socioeconômica}

Para avaliar o nível socioeconômico, foi utilizada a metodologia proposta por Kozlowski ${ }^{27}$ e Meneghim et al..$^{28}$, que classifica os sujeitos em seis classes socioeconômicas, a partir da ponderação de cinco fatores: renda familiar, número de pessoas na família, grau de instrução dos pais, tipo de habitação e profissão do responsável. Os cinco fatores recebem uma pontuação cujo somatório possibilita a determinação de um escore individual, que varia de 10,0 a 100,0 e permite a hierarquização dos sujeitos dentro de seis classes sociais: baixa inferior $(\mathrm{F})$; baixa $(\mathrm{E})$; média inferior $(\mathrm{D})$; média $(\mathrm{C})$; média superior $(\mathrm{B})$ e alta $(\mathrm{A})$.

\section{Procedimentos}

Em primeiro lugar, os pais assinavam o Termo de Consentimento Livre e Esclarecido e a seguir respondiam aos questionários. Só então as crianças eram avaliadas, por um único examinador, previamente calibrado (KAPPA > 0,87), segundo calibração intraexaminadores padronizado pela $\mathrm{OMS}^{45}$ e auxiliado por um anotador, em local que permitisse o máximo aproveitamento de luz natural. O registro foi realizado em odontograma próprio e individual para cada criança ${ }^{45}$.

\section{Tratamento dos Dados}

Primeiramente, foi realizada a análise descritiva dos dados, de posição e variabilidade. $\mathrm{Na}$ medida em que se observou a normalidade das variáveis, através do teste de Shapiro Wilk, para comparação de médias utilizou-se o ANOVA para grupos independentes, seguido do teste de comparações múltiplas de Tukey, quando houve diferenças estatisticamente significativas. Finalmente, aplicou-se um modelo de regressão logística binária, tendo como variável desfecho ceo-d $>5$ e como variáveis explicativas o nível socioeconômico, Lócus de Controle, Lócus de Controle Parental e atitudes parentais.

O programa usado para as análises foi o Statistical Package for the Social Sciences for Windows. Os resultados foram discutidos no nível de $5 \%$ de significância.

\section{Resultados}

A leitura da Tabela 1 permite verificar que houve grande discrepância entre o número de participantes de entidades públicas e privadas. Das 426 crianças avaliadas, 389 (91,31\%) estavam matriculadas nas creches municipais e somente 37 (8,69\%), nas escolas particulares. Em relação ao sexo, a distribuição foi mais uniforme, pouco mais da metade da amostra era composta por meninos (53,05\%). Quanto à avaliação do ceo-d, $47,65 \%$ das crianças apresentaram-se livres de cárie, 33,33\% tinham, no máximo, quatro lesões de cárie e os 19,01\% alunos restantes apresentavam cinco ou mais dentes com cárie.

$\mathrm{Na}$ Tabela 2 pode-se observar grande diferen- 
ça na porcentagem de dentes com cárie das crianças de escolas públicas e privadas, especialmente com relação à ausência de cárie e ao ceo-d 5 ou mais. Quanto ao índice socioeconômico, sua relação com prevalência de cárie foi altamente significativa ( $\mathrm{p}<0,001)$ : uma porcentagem maior de crianças das classes A, B e C estavam livres de cárie $(64,20 \%)$ quando comparadas com as crianças das classes D $(48,84 \%)$ e E-F $(40,74 \%)$. Foi, também, nas classes sociais E-F que se encontrou o maior percentual de crianças com ceo -d maior que 5.

Quanto ao Lócus de Controle dos pais, pais com filhos com ceo-d 1 a 4 tinham, de forma significativa, uma média menor de Acaso no Lócus de Controle (ALC), isto é, acreditavam mais no acaso como responsável pela doença do que os pais de filhos sem cárie. Quando se avaliou o Lócus de Controle parental, pais com média mais baixa na dimensão Internalidade no Lócus de Controle (ILC), tinham, de forma altamente significativa, filhos com menos cárie $(\mathrm{p}<0,0001)$.

Tabela 1. Distribuição das crianças segundo tipo de escola, sexo e índice ceo-d.

\begin{tabular}{lrr}
\hline Escola & N & \% \\
\cline { 2 - 3 } Pública & 389 & 91,31 \\
Privada & 37 & 8,69 \\
Sexo & & \\
Masculino & 226 & 53,05 \\
Feminino & 200 & 46,95 \\
Ceo-d & & \\
Livre de Cárie & 203 & 47,65 \\
Ceo-d até 4 & 142 & 33,33 \\
\hline
\end{tabular}

Por outro lado, pais com médias mais baixas na dimensão Externalidade no Lócus de Controle (ELC), que atribuíam mais a fatores externos (outros e poderosos) à saúde do filho, tinham mais filhos com 1 a 4 dentes com cárie. (Tabela 3)

Ao associar o Lócus de Controle e as atitudes parentais com variáveis sociodemográficas, a dimensão Externalidade e Acaso se associaram, significativamente, com nível socioeconômico $(0,017)$. As atitudes parentais, por sua vez, tiveram uma associação significativa com nível socioeconômico $(<0,0001)$, pais pertencentes às classes $\mathrm{A}-\mathrm{B}-\mathrm{C}$ verbalizaram atitudes mais adequadas que aqueles que faziam parte das D-E-F.

Na regressão logística, apresentada na Tabela 4, maior índice socioeconômico e baixa externalidade parental apareceram como fatores de proteção e a baixa internalidade mostrou-se como fator de risco para crianças com ceo-d maior ou igual a 5. Pais que atribuíam menos o controle da doença dos filhos a outros poderosos (P) tinham $85 \%$ mais chance de não ter filhos com maior número de cárie. Por outro lado, pais que acreditavam pouco que o controle das doenças do filho estava sob seu controle tinham 33\% mais chance de ter filhos com cinco ou mais dentes cariados.

\section{Discussão}

A prevalência de crianças com cárie desta amostra foi superior ao estipulado pela Organização Mundial de Saúde - OMS, para ser alcançado até o ano 2000, de 50\% das crianças entre 5 e 6 anos livres de cárie ${ }^{43}$. Foi superior, também, à de outros estudos com pré-escolares do estado de São Paulo, em que, aproximadamente, 50 a $55 \%$ das

Tabela 2. Comparação entre os índices de ceo-d de crianças que frequentavam escolas públicas e particulares e pertencentes a diferentes classes socioeconômicas.

\begin{tabular}{|c|c|c|c|c|c|c|c|c|c|c|c|}
\hline \multirow{3}{*}{ Variável } & \multirow{3}{*}{ Categorias } & \multicolumn{8}{|c|}{ Ceo-d } & \multirow{3}{*}{$x^{2}$} & \multirow{3}{*}{$\mathbf{p}$} \\
\hline & & \multicolumn{2}{|r|}{$\mathbf{0}$} & \multicolumn{2}{|c|}{ De 1 a 4} & \multicolumn{2}{|c|}{5 ou mais } & \multicolumn{2}{|c|}{ Total } & & \\
\hline & & $\mathbf{n}$ & $\%$ & $\mathbf{n}$ & $\%$ & $\mathbf{N}$ & $\%$ & $\mathbf{n}$ & $\%$ & & \\
\hline \multirow{5}{*}{ Sócio Econômico } & Pública & $178^{\mathrm{a}}$ & 45,76 & $130^{\mathrm{a}}$ & 33,42 & $81^{\mathrm{a}}$ & 20,82 & 389 & 100,0 & 11,09 & 0,0039 \\
\hline & Particular & $25^{\mathrm{b}}$ & 67,57 & $12^{\mathrm{a}}$ & 32,43 & $0^{\mathrm{b}}$ & 0,00 & 37 & 100,0 & & \\
\hline & $E-F$ & $88^{\mathrm{a}}$ & 40,74 & $74^{\mathrm{a}}$ & 34,26 & $54^{\mathrm{a}}$ & 25,00 & 216 & 100,0 & & \\
\hline & $\mathrm{D}$ & $63^{\mathrm{a}}$ & 48,84 & $45^{\mathrm{a}}$ & 34,88 & $21^{\mathrm{ac}}$ & 16,28 & 129 & 100,0 & 17,91 & 0,001 \\
\hline & A-B-C & $52^{\mathrm{b}}$ & 64,20 & $23^{\mathrm{a}}$ & 28,39 & $6^{\mathrm{bc}}$ & 7,41 & 81 & 100,0 & & \\
\hline
\end{tabular}

$(\mathrm{p}<0,05){ }^{*}$ Proporções seguidas de mesma letra não diferem significativamente pelo teste de proporções ao nível de 5\% para a categoria ceo-d. 


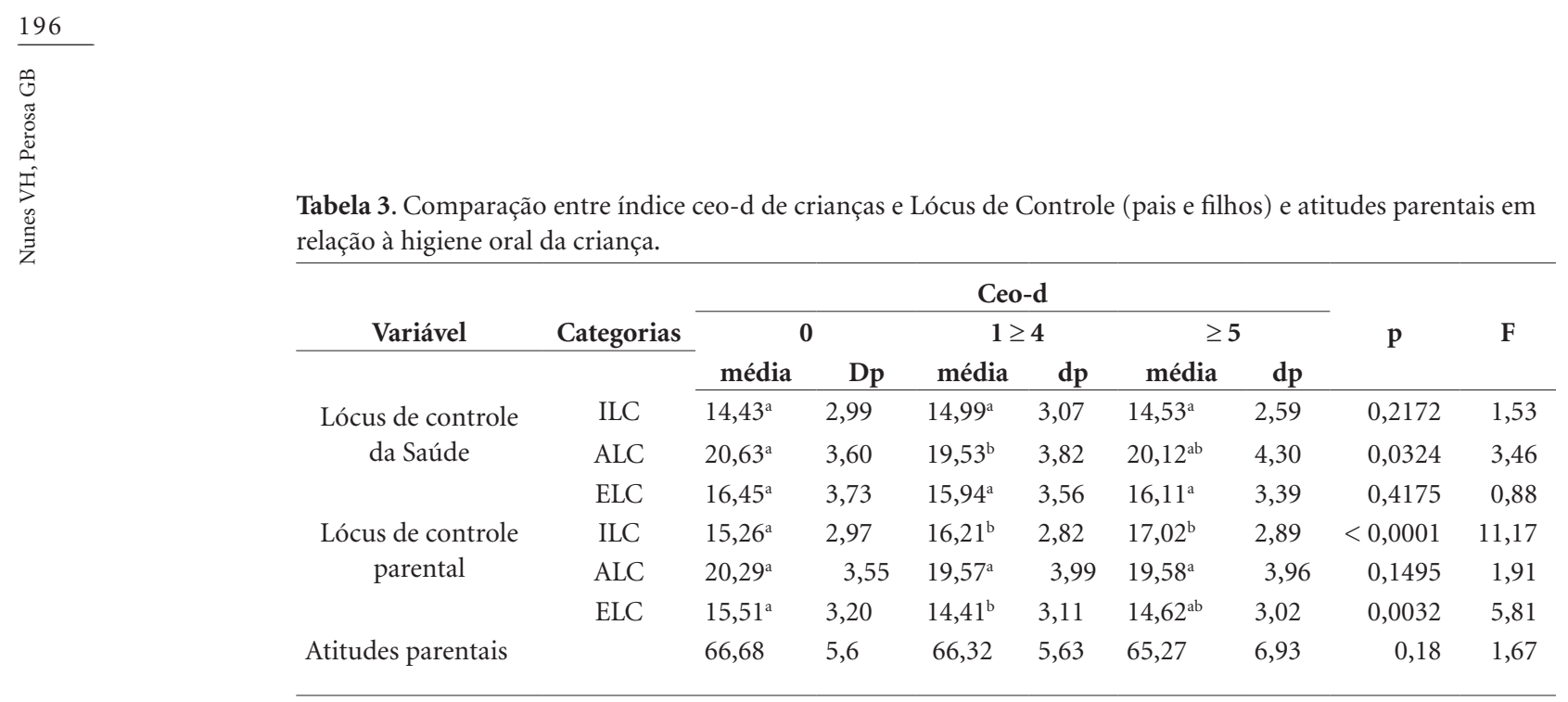

*Médias seguidas de mesma letra não diferem significativamente pelo teste de Tukey ao nível de 5\%.

Tabela 4. Modelo de Regressão Logística para explicar risco para ceo-d maior ou igual a 5.

\begin{tabular}{lccccc}
\hline \multicolumn{1}{c}{ Variável } & Categorias & Estimativa & Erro & $\mathbf{p}$ & OR (IC95\%) \\
\hline Constante & 1 & -2.8197 & 1.6656 & 0.0905 & - \\
Socioeconômico & 2 & -1.0273 & 1.6591 & 0.5358 & - \\
& E $-\mathrm{F}$ & - & - & - & 1 \\
Lócus de Controle Pais & $\mathrm{D}$ & 0.1623 & 0.1533 & 0.2896 & $0.761(0.478-1.212)$ \\
& A $-\mathrm{B}-\mathrm{C}$ & -0.5975 & 0.1888 & 0.0016 & $0.356(0.196-0.647)$ \\
& ILC & -0.0765 & 0.0432 & 0.0764 & $0.926(0.851-1.008)$ \\
Lócus de Controle Filhos & ALC & 0.0197 & 0.0404 & 0.6263 & $1.020(0.942-1.104)$ \\
& ELC & 0.0184 & 0.0395 & 0.6415 & $1.019(0.943-1.101)$ \\
& ILC & 0.2838 & 0.0470 & $<.0001$ & $1.328(1.211-1.456)$ \\
Atitudes Parentais & ALC & -0.0233 & 0.0398 & 0.5577 & $0.977(0.904-1.056)$ \\
& ELC & -0.1682 & 0.0456 & 0.0002 & $0.845(0.773-0.924)$ \\
& Atitude & -0.00158 & 0.0191 & 0.9340 & $0.998(0.962-1.036)$
\end{tabular}

crianças estavam livres de cárie ${ }^{8-10}$. A diversidade de resultados talvez se deva à idade dos sujeitos, a diferenças metodológicas, mas, principalmente, a ações preventivas e de planejamento de ações odontológicas muito diversas nas cidades em que se realizaram as pesquisas. Em Paulínia ${ }^{9}$ e em outras cidades do interior paulista ${ }^{46}$ ocorrem levantamentos epidemiológicos de rotina para diagnosticar as condições de saúde bucal das populações, contribuindo para um planejamento mais direcionado dos serviços de saúde ${ }^{9,47}$, assim como há monitoramento contínuo das condições bucais das crianças. Segundo Baldani et al. $^{48}$, para facilitar o monitoramento e a adesão aos programas de atenção em saúde bucal, tanto curativos, preventivos como educativos, eles precisam ocorrer em contextos que atendam crianças pequenas, como na Estratégia de Saúde da Família -ESF, nos centros de saúde, onde vão para as consultas de Puericultura e nas creches e pré-escolas. Nas creches do município onde se realizou este estudo não havia uma programação de ações preventivas junto aos escolares dessa faixa etária ou atendimento odontológico de rotina.

Confirmando dados de literatura ${ }^{49-52}$ houve uma porcentagem mais alta de cárie severa nos extratos socioeconômicos mais baixos, com uma maior concentração de crianças com ceo-d maior que 5 nos níveis E-F. Coerentemente, 67,57\% crianças que frequentavam escolas particulares estavam livres de cárie, não foi encontrada nenhuma ocorrência de ceo-d maior ou igual a cinco, enquanto que das crianças matriculadas nas CEI's municipais, $45,76 \%$ estavam livres de cárie e $20,82 \%$ tinham ceo-d maior ou igual a cinco. Para Pauleto et al. ${ }^{46}$, a saúde bucal e o de- 
senvolvimento da cárie ocorrem pela junção de influências distintas associadas ao nível socioeconômico: renda familiar, condições ambientais, consumo de alimentos, hábitos de higiene, acesso a serviços de saúde e informação. O tipo de dieta, por exemplo, difere nas diferentes camadas sociais ${ }^{16,17}$. Tomita et al. ${ }^{17}$ observaram que as crianças de estratos sociais mais baixos tinham preferência pelo sabor mais adocicado, o que pode explicar o maior consumo de açúcar entre as crianças de menor renda, um dos fatores que predispõe à cárie ${ }^{49}$. Por outro lado, identificou-se que grupos com maior nível de educação paterna, tinham maior consciência dos efeitos prejudiciais do açúcar ${ }^{53}$. Nas populações mais pobres, verificou-se, também, menor procura pelos serviços de saúde, especialmente dos preventivos ${ }^{54}$.

Em relação às atitudes frente à saúde bucal, os pais, especialmente os de escolaridade superior e maior nível socioeconômico, mostraram um alto índice de conhecimento sobre atitudes corretas. Entretanto, contrariando a literatura ${ }^{41,55}$, o nível de conhecimentos não se associou com o índice de cárie das crianças. Possivelmente os pais podem ter conhecimentos adequados, mas eles não se refletem em sua prática diária. Na revisão bibliográfica de Castilho et al. ${ }^{54}$ comprovou-se que os comportamentos dos pais têm maior impacto específico sobre a saúde bucal da criança que seus conhecimentos e atitudes.

Quando se avaliou o Lócus de Controle sobre a própria saúde observou-se que os pais que acreditavam no acaso tinham filhos com mais cárie $(\mathrm{p}<0,032)$. De forma significativa, acreditar no acaso associou-se ao menor nível socioeconômico. Rodríguez-Rosero et al..$^{55}$ também observaram que uma porcentagem maior de mães que não haviam cursado ensino superior acreditavam mais que outros agentes, ou mesmo o acaso, controlava sua saúde, quando comparadas com mães com maior escolaridade. Ao se aplicar o instrumento adaptado, avaliando o Lócus de Controle parental, confirmando resultados de outros estudos $^{19,56}$, crianças com menor índice de cárie tinham, de forma altamente significativa, pais que acreditavam que suas ações influenciavam na saúde de seus filhos ( $\mathrm{p}<0,0001)$ e, por outro lado, pais que atribuíam a saúde do filho a fatores externos, tinham filhos com 1 a 4 dentes com cárie.

$\mathrm{Na}$ regressão final, baixa internalidade se mostrou como fator de risco para ceo-d maior ou igual a 5 e, coerentemente, baixa externalidade como fator de proteção. Esses achados reforçam a hipótese que as expectativas de controle influenciam os comportamentos que a mãe emite para propiciar o desenvolvimento da criança e que a percepção parental de controle interno sobre a saúde do filho pode favorecer cuidados preventivos e, consequentemente, o nível de cárie da criança ${ }^{32}$.

As diferenças obtidas a partir da aplicação dos dois instrumentos, o original de Dela Coleta ${ }^{44}$ e o adaptado, reforça a importância do uso de instrumentos específicos na investigação das crenças e das atitudes paternas. Cerqueira e Nascimento ${ }^{32}$ já alertavam que os efeitos das expectativas parentais são mais claramente compreendidos ao se investigar, especificamente, a expectativa do cuidador, em relação à saúde da criança. Se ao se posicionar quanto ao que controlava sua saúde, majoritariamente, os pais referiram que eram suas próprias ações, ao avaliar seu papel na saúde do filho os pais acreditavam que, em primeiro lugar, as ações de outros, incluindo a própria criança, tinha maior interferência no seu processo saúde/ doença. Ao atribuir à criança parte da responsabilidade sobre sua saúde, os pais parecem compartilhar da crença que ela é um ser dinâmico, não passivo apenas às exigências de seu meio ${ }^{57}$.

A concepção de criança como um ser ativo modificou as práticas educativas das famílias brasileiras nos últimos anos, com um deslocamento nas relações de autoridade pais/filhos, passando de um modelo baseado na imposição e no controle para outro fundamentado na participação e na negociação, com crescente valorização das iniciativas da criança, por acreditar que, além das ações paternas, sua individualidade interfere em sua socialização ${ }^{58,59}$.

No entanto, para alguns autores, a maior participação da criança na determinação de seu cotidiano vem criando conflitos em muitos pais, gerando grande insegurança, dificuldade em colocar limites, culpa ao tomar atitudes restritivas e, principalmente, muitas dúvidas quanto à real competência em saber cuidar de uma criança que desobedece e não segue as recomendações paternas ${ }^{59,60}$. Possivelmente os pais desta amostra, mesmo os que acreditavam que as próprias ações determinam a sua condição de saúde, ao tentar introduzir hábitos saudáveis para o filho, tenham encontrado resistências e dificuldades, avaliando que não possuíam as competências requeridas para esse fim, abalando sua percepção de autoeficácia ${ }^{60}$, sua crença no controle, um forte determinante da manutenção de ações preventivas eficazes e de comportamentos de cuidado em saúde ${ }^{34,60}$. Esse fato poderia explicar porque, apesar dos pais possuírem os conhecimentos es- 
senciais sobre cuidados bucais, ele não se refletiu no nível de cárie. No estudo de Adair et al. ${ }^{61}$ a baixa percepção de autoeficácia dos pais fazia com que, apesar de conhecer as atitudes adequadas em saúde oral e reconhecer sua responsabilidade, eles não tomassem medidas preventivas para garantir hábitos de escovação bidiária e consumo de alimentos açucarados.

Algumas limitações do estudo exigem cautela na generalização dos resultados. Em primeiro lugar, eles foram obtidos a partir de uma amostra de conveniência que apesar de ter alta representatividade de pré-escolares matriculados em escolas públicas, não obteve o mesmo sucesso em relação a alunos de escolas particulares, onde se registrou o maior número de perdas. Outras pesquisas de saúde bucal em pré-escolares também tiveram baixa representatividade de alunos de escolas particulares ${ }^{27}$ ou se limitaram a avaliar crianças de rede pública ${ }^{2,5,41}$, possivelmente porque há um número menor de crianças desta faixa etária, cujos pais têm melhor renda, frequentando escola ou pela dificuldade de contar com o consentimento de alunos de rede privada nas pesquisas. Em relação aos instrumentos, os índices de precisão foram baixos, com coeficientes alfa de Cronbach $(\alpha)$ inferiores a 0,60 , tanto no questionário traduzido e adaptado por Dela Coleta $^{44}$, como no instrumento adaptado neste trabalho para avaliar o Lócus de Controle Paren- tal. Segundo Pasquali et al. ${ }^{62}$, apesar da expansão de pesquisas sobre lócus de controle, ele é um constructo complexo, que ainda precisa de melhor operacionalização e maior número de itens nos questionários para ser avaliado a contento.

\section{Conclusão}

Neste estudo, que avaliou $80,22 \%$ do total dos alunos de 5 anos matriculados nas pré-escolas de Avaré-SP, houve associação entre a presença de cárie nas crianças, o nível socioeconômico e o Lócus de Controle parental. O Lócus de Controle parental, em sua dimensão baixa internalidade, quando o cuidador não se percebe como principal determinante do processo saúde/doença de seu filho, se mostrou um fator de risco para cárie nos dentes decíduos, possivelmente porque a mãe espera a ação de outros, retardando cuidados que podem proteger de adoecimentos ou atenuar sua gravidade. A associação do nível de carie das crianças com a alta externalidade materna, quando a mãe acredita que outros, incluindo a própria criança, também controlam o processo saúde doença do filho, sugere a necessidade que, em próximos estudos, se investigue, além do Lócus de Controle, a percepção de autoeficácia materna e sua confiança em levar o filho a adquirir hábitos bucais saudáveis.

\section{Colaboradores}

VH Nunes e GB Perosa participaram na concepção do estudo, revisão da literatura, definição do desenho metodológico, processamento e análise dos dados, discussão dos resultados, redação e revisão crítica do texto. 


\section{Referências}

1. Fisher-Owens SA, Gansky SA, Platt LJ, Weintraub JA Soobader MJ, Bramlett MD, Newacheck PW. Influences on children's oral health: a conceptual model. Pediatrics 2007; 120(3):e510-e520.

2. Brandão IMG, Arcieri RM, Sundefeld MLM, Moimaz. SAS Cárie precoce: influência de variáveis sócio-comportamentais e o locus de controle da saúde em um grupo de crianças de Araraquara, São Paulo, Brasil. Cad Saude Publica 2006; 22(6):1247-1256.

3. American Academy of Pediatric Dentistry. Clinical guideline on baby bottle tooth decay/early childhood caries/breastfeeding/early childhood caries: unique challenges and treatment options. [cited 2013 Aug 1]. Available from: http://www.aapd.org/members/referencemanual/pdfs/Baby_Bottle_TDEEC.pdf

4. Casamassimo PS. Relationships between oral and systemic health. Pediatr Clin North Am 2000; 47(5):11491157.

5. Low W, Tan S, Schwartz S. The effect of severe caries on the quality of life in young children. Pediatr Dent 1999; 21(6):325-326.

6. Colares V, Feitosa S. O desempenho na pré-escola de crianças portadoras de carie severa. Acta Sci Health Sci 2003; 25(2):129-134.

7. Brasil. Ministério da Saúde (MS). Secretaria de Atenção à Saúde. Secretaria de Vigilância em Saúde. SB Brasil 2010: Pesquisa Nacional de Saúde Bucal: resultados principais. Brasília: MS; 2012.

8. Dini EL, Holt RD, Bedi R. Caries and its association with infant feeding and oral health related behaviours in 3-4 year old Brazilian children. Community Dent Oral Epidemiol 2000; 28(4):241-248.

9. Gomes PR, Costa SC, Cypriano S, Sousa MLR. Paulínia, São Paulo, Brasil: situação da cárie dentária com relação às metas OMS 2000 e 2010. Cad Saude Publica 2004; 20(3):866-870.

10. Tagliaferro EPDS, Ambrosano GMB, Meneghim MDC, Pereira AC. Risk indicators and risk predictors of dental caries in schoolchildren. J Appl Oral Sci 2008; 16(6):408-413.

11. Bardal PAP, Olympio KPK, Valle AAD, Tomita NE. Cárie dentária em crianças como fenômeno natural ou patológico: ênfase na abordagem qualitativa. Cien Saude Colet 2006; 11(1):161-167.

12. Almeida TF, Vianna MI, Cabral MB, Cangussu MC, Floriano FR. Family context and incidence of dental caries in preschool children living in areas covered by the Family Health Strategy in Salvador, Bahia State, Brazil. Cad Saude Publica 2012; 28(6):1183-1195.

13. Acharya S, Pentapati KC, Singh S. Influence of socioeconomic status on the relationship between locus of control and oral health. Oral Health Prev Dent 2011; 9(1):9.

14. Aleksejūnienè J, Brukienè V. Parenting style, locus of control, and oral hygiene in adolescents. Medicina (Kaunas) 2012; 48(2):102-108.

15. Prakash P, Subramaniam P, Durgesh BH, Konde S. Prevalence of early childhood caries and associated risk factors in preschool children of urban Bangalore, India: a cross-sectional study. Eur J Dent 2012; 6(2):141-152.

16. Novais SMA, Batalha RP, Grinfeld S, Fortes TM, Pereira MAS. Relação doença cárie-açúcar: prevalência em crianças. Pesq Bras Odontoped Clin Integr 2004; 4(3):199-203.
17. Tomita NE, Nadanovsky P, Vieira ALF, Lopes ES. Preferências por alimentos doces e cárie dentária em pré-escolares. Rev Saude Publica 1999; 33(6):542-546.

18. Granville-Garcia AF, Menezes VA. Experiência de cárie em pré-escolares da rede publica e privada da cidade de Recife - PE. Pesqui. Bras. Odontopediatr. Clin. Integr. 2005; 5(2):103-109.

19. Lenčová E, Pikhart H, Broukal Z, Tsakos G. Relationship between parental locus of control and caries experience in preschool children - cross-sectional survey. BMC Public Health 2008; 8:208.

20. Freire MDCM, Sheiham A, Bino YA. Hábitos de higiene bucal e fatores sociodemográficos em adolescentes. Rev bras epidemiol 2007, 10(4):606-614.

21. Barros AJ, Bertoldi AD. Desigualdades na utilização e no acesso a serviços odontológicos: uma avaliação em nível nacional. Cien Saude Colet 2002; 7(4):709-717.

22. Pattussi MP, Marcenes W, Croucher R, Sheiham A. Social deprivation, income inequality, social cohesion and dental caries in Brazilian school children. Soc Sci Med 2001; 53(7):915-925.

23. Wood NS, Costeloe K, Gibson AT, Hennessy EM, Marlow N, Wilkinson AR. The EPICure study: growth and associated problems in children born at 25 weeks of gestational age or less. Arch Dis Child Fetal Neonatal 2003; 88(6):F492-F500

24. Pickett KE, Mookherjee J, Wilkinson RG. Teenage births and violence are related to income inequality among rich countries. Am J Public Health 2005; 95(7):1181-1183.

25. Von Rueden U, Gosch A, Rajmil L, Bisegger C, Ravens-Sieberer U. Socioeconomic determinants of health related quality of life in childhood and adolescence: results from a European study. J Epidemiol Community Health 2006; 60(2):130-135.

26. Tomita NE, Sheiham A, Bijella VT, Franco LJ. Relação entre determinantes socioeconômicos e hábitos bucais de risco para más-oclusões em pré-escolares The relationship between socioeconomic determinants and oral habits as risk factors for malocclusion in preschool children. Pesqui. Odontol. Bras. 2000; 14(2):169-175.

27. Kozlowski FC. Relação entre o fator socioeconômico e a prevalência e severidade de fluorose e cárie dentária [tese]. Piracicaba: Universidade Estadual de Campinas; 2001.

28. Meneghim MC, Kozlowski FC, Pereira AC, Ambrosano GMB, Meneghim ZMAP. Classificação socioeconômica e sua discussão em relação à prevalência de cárie e fluorose dentária. Cien Saude Colet 2007; 12(2):523-529.

29. Yunes MAM. Psicologia positiva e resiliência: o foco no indivíduo e na família. Psicol. Estud. 2003; 8(esp.):7584.

30. Engle PL. Maternal mental health: program and policy implications. J Clin Nutr 2009; 89(3):963-970.

31. Lordelo EDR, Fonseca AL, Araújo MLVD. Responsividade do ambiente de desenvolvimento: crenças e práticas como sistema cultural de criação de filhos. Psicol. Reflex. Crit. 2000; 13(1):73-80.

32. Cerqueira MMMD, Nascimento ED. Construção e validação da Escala de Lócus de Controle Parental na Saúde. Psico USF 2008; 13(2):253-263.

33. Kobarg AP, Sachetti A, Vieira ML. Valores e crenças parentais: reflexões teóricas. Rev Bras. Crescimento Desenvolv. Humano 2006; 16(2):96-102. 
34. Bandura A. Regulation of Cognitive Processes Through Perceived Self-Efficacy. Developmental Psychology 1989; 25(5):729-735.

35. Costa VSG. Crenças e atitudes parentais: relação com os comportamentos preventivos e com a cárie precoce da infância [dissertação]. Lisboa: Universidade de Lisboa; 2011.

36. Peker K, Uysal Ö, Bermek G. Dental training and changes in oral health attitudes and behaviors in Istanbul dental students. J Dent Educ 2010; 74(9):1017-1023.

37. Tinsley BJ, Holtgrave DR. Maternal health locus of control beliefs, utilization of childhood preventive health services, and infant health. J Dev Behav Pediatr 1989; 10(5):236-241.

38. Chase I, Berkowitz RJ, Mundorff S, Proskin H, Weinstein P, Billings R. Clinical outcomes for early childhood caries: the influence of salivary mutans streptococci levels. Eur J Pediatr Dent 2004; 5(3):143-146.

39. Wallston KA, Wallston BS, Devellis R. Development of the Multidimensional Health Locus of Control (MHLC) Scales. Health Educ Monogr 1978; 6(2):160170.

40. Dumitrescu AL, Wagle M, Dogaru BC, Manolescu B. Modeling the theory of planned behavior for intention to improve oral health behaviors: the impact of attitudes, knowledge, and current behavior. J Oral Sci 2011; 53(3):369-377.

41. Navas RP, Morales TR, Zambrano O, Álvarez CJ, Santana Y, Viera N. salud bucal en preescolares: su relación con las actitudes y nivel educativo de los padres Interciencia 2002; 27(11):631-634.

42. Instituto Brasileiro de Geografia e Estatística (IBGE). Censo demográfico 2010: características da população e dos domicílios: resultados do universo. Rio de Janeiro: IBGE; 2010.

43. Brasil. Ministério da Saúde (MS). Secretaria de Atenção à Saúde. Departamento de Atenção Básica. Coordenação Nacional de Saúde Bucal. Projeto SB Brasil 2003: condições de saúde bucal da população brasileira 20022003. Brasília: MS; 2004.

44. Dela Coleta MF. O modelo de crenças em saúde: uma aplicação a comportamentos de prevenção e controle da doença cardiovascular [tese]. Brasília: Universidade de Brasília; 1995.

45. World Health Organization (WHO). Oral health surveys: basic methods. $4^{\text {th }}$ ed. Geneva: ORH, EPID; 1997.

46. Pauleto ARC, Pereira MLT, Cyrino EG. Oral health: a critical review about educative programmes for students. Cien Saude Colet 2004; 9(1):121-130.

47. Hallett KB, O’Rourke PK. Social and behaviour determinants of early childhood caries. Aust Dent J 2003; 48(1):27-33.

48. Baldani MH, Fadel CB, PossamaI T, Queiroz MGS. A inclusão da odontologia no Programa Saúde da Família no Estado do Paraná, Brasil Inclusion of oral health services in the Family Health Program in the State of Paraná, Brazil. Cad Saude Publica 2005; 21(4):10261035.
49. Harris R, Nicoll AD, Adair PM, Pine CM. Risk factors for dental caries in young children: a systematic review of the literature. Community Dent Health 2004; 21(1 Supl.):71-85.

50. Peres MA, Latorre MRDO, Sheiham A, Peres KGA, Barros FC, Hernandez PG, Mass AMN, Romano AR, Victoria CG. Social and biological early life influences on severity of dental caries in children aged 6 years. Community Dent Oral Epidemiol 2005; 33(1):53-63.

51. Schroth RJ, Moffatt MEK. Determinants of early childhood caries (ECC) in a Rural Manitoba Community: a pilot study. Pediatr Dent 2005; 27(2):114-120.

52. Aquino RC, Philippi ST. Consumo infantil de alimentos industrializados e renda familiar na cidade de São Paulo. Rev Saude Publica 2002; 36(6):655-606.

53. Kassouf AL. Acesso aos serviços de saúde nas áreas urbana e rural do Brasil. Rev Econ. Sociol. Rural 2005; 43(1):29-44.

54. Castilho ARFD, Mialhe FL, Barbosa TDS, Puppin-Rontani RM. Influence of family environment on children's oral health: a systematic review. J Pediatr 2013; 89(2):116-123.

55. Rodríguez-Rosero JE, Ferriani MDGC, Dela Coleta MF Escala de lócus de controle da saúde-MHLC: estudos de validação. Rev. Latino-am. Enferm. 2002; 10(2):179184 .

56. Reisine S, Litt M. Social and psychological theories and their use for dental practice. Int Dent J 1993; 43(3Supl. 1):279-287

57. Sameroff A. A unified theory of development: a dialectic integration of nature and nurture. Child Dev 2010; 81(1):6-22.

58. Biasoli-Alves ZMM. Famílias brasileiras do século XX: os valores e as práticas de educação da criança. Temas Psicol 1997; 5(3):33-49.

59. Montandon C. As práticas educativas parentais e a experiência das crianças. Educação e Sociedade 2005; 26(91):485-507.

60. Zagury T. Limites e conflitos na adolescência. Zagury T. Educar sem culpa: a gênese da ética. $6^{a}$ ed. Rio de Janeiro: Record; 1995. p. 81-93.

61. Adair PM, Pine CM, Burnside G, Nicoll AD, Gillett A, Anwar S, Young DW. Familial and cultural perceptions and beliefs of oral hygiene and dietary practices among ethnically and socio-economically diverse groups. Commun Dent Health 2004; 21(1):102-111.

62. Pasquali L, Alves AR, Pereira MA. Scale of Locus of control-ELCO. Psicologia: Reflexão e Crítica 1998; 11(2):363-378.

Artigo apresentado em 16/03/2015

Aprovado em 01/10/2015

Versão final apresentada em 03/10/2015 
ERRATA ERRATUM

p. 191

onde se lê:

monitoring points

leia-se:

locus of control 